\title{
Optimal Siting and Sizing of Energy Storage System for Power Systems with Large- scale Wind Power Integration
}

Zhao, Haoran; Wu, Qiuwei; Huang, Shaojun; Guo, Qinglai ; Sun, Hongbin; Xue, Yusheng

Published in:

Proceedings of 2015 IEEE PowerTech.

Link to article, DOI:

10.1109/PTC.2015.7232615

Publication date:

2015

Document Version

Peer reviewed version

Link back to DTU Orbit

Citation (APA):

Zhao, H., Wu, Q., Huang, S., Guo, Q., Sun, H., \& Xue, Y. (2015). Optimal Siting and Sizing of Energy Storage System for Power Systems with Large-scale Wind Power Integration. In Proceedings of 2015 IEEE PowerTech. IEEE. https://doi.org/10.1109/PTC.2015.7232615

\section{General rights}

Copyright and moral rights for the publications made accessible in the public portal are retained by the authors and/or other copyright owners and it is a condition of accessing publications that users recognise and abide by the legal requirements associated with these rights.

- Users may download and print one copy of any publication from the public portal for the purpose of private study or research.

- You may not further distribute the material or use it for any profit-making activity or commercial gain

- You may freely distribute the URL identifying the publication in the public portal 


\section{Optimal Siting and Sizing of Energy Storage System for Power Systems with Large-scale Wind Power Integration}

\author{
Haoran Zhao, Qiuwei Wu, Shaojun Huang \\ Center for Electrical Power and Energy \\ Technical University of Denmark \\ Lyngby, Denmark \\ \{hzhao,qw,shuang\}@elektro.dtu.dk
}

Qinglai Guo, Hongbin Sun

Department of Electrical Engineering

Tsinghua University

Beijing, China

\{guoqinglai,shb\}@mail.tsinghua.edu.cn \{xueyusheng\}@ sgepri.sgcc.com.cn

\section{Scalar variables}

$P_{i}^{g}(k) \quad$ Power of conventional generator of Bus $i$ at time step $k, i \in \mathcal{G}_{g}$.

$P_{i}^{r}(k) \quad$ Power of renewable energy generator of Bus $i$ at time step $k, i \in \mathcal{G}_{r}$.

$P_{i}^{s}(k) \quad$ Power of ESS unit of Bus $i$ at time step $k, i \in \mathcal{S}$.

$P_{i}^{l}(k) \quad$ Load demand of Bus $i$ at time step $k, i \in \mathcal{N}$.

$\Delta P_{i}^{g}(k)$ Ramp rate of $P_{i}^{g}(k)$.

$\Delta P_{i}^{s}(k)$ Ramp rate of $P_{i}^{s}(k)$.

$P_{\text {loss }} \quad$ Transmission losses of network.

\begin{abstract}
This paper proposes algorithms for optimal siting and sizing of Energy Storage System (ESS) for the operation planning of power systems with large scale wind power integration. The ESS in this study aims to mitigate the wind power fluctuations during the interval between two rolling Economic Dispatches (EDs) in order to maintain generation-load balance. The charging and discharging of ESS is optimized considering operation cost of conventional generators, capital cost of ESS and transmission losses. The statistics from simulated system operations are then coupled to the planning process to determine the optimal siting and sizing of storage units throughout the network. These questions are investigated using an IEEE benchmark system.
\end{abstract}

Index Terms-Wind power, ESS, optimal siting, optimal sizing.

\section{NOMENCLATURE}

\section{A. Parameters}

$N_{b} \quad$ Number of buses.

$N_{l} \quad$ Number of transmission lines.

$N_{g} \quad$ Number of conventional generators.

$N_{r} \quad$ Number of renewable energy generators.

$N_{s} \quad$ Number of ESS units.

$M \quad$ Number of time steps.

$\alpha_{1}, \alpha_{2}, \alpha_{3}$

Weighting factors of cost function.

$g_{i j} \quad$ Conductance of the transmission line between Bus $i$ and Bus $j$.

\section{B. Sets}

$\mathcal{N} \quad$ Set of all buses, $\mathcal{N}=\left\{1, \cdots, N_{b}\right\}$.

$\mathcal{L} \quad$ Set of transmission lines, $\mathcal{L}=\left\{1, \cdots, N_{l}\right\}$.

$\mathcal{G}_{g} \quad$ Set of conventional generator buses, $\mathcal{G}_{g}=$ $\left\{1, \cdots, N_{g}\right\}$.

$\mathcal{G}_{r} \quad$ Set of renewable energy generator buses, $\mathcal{G}_{g}=$ $\left\{1, \cdots, N_{r}\right\}$.

$\mathcal{G} \quad$ Set of all generator buses, $\mathcal{G}=\mathcal{G}_{g} \cup \mathcal{G}_{r}$.

$\mathcal{S} \quad$ Set of ESS buses, $\mathcal{S}=\left\{1,2, \cdots, N_{s}\right\}$.

The work was supported by the Sino-Danish Centre for Education and Research (SDC) throuth the PhD project of "Coordinated Control of Wind Power Plants and Energy Storage Systems".

\section{Matrix variables}

$P \quad$ Vector of active power injections for all the buses $\mathcal{N}, P \in \mathbb{R}^{N_{b} \times 1}$.

$B \quad$ Admittance matrix neglecting the resistance, $B \in$ $\mathbb{R}^{N_{b} \times N_{b}}$

$F \quad$ Vector of line flows for all the transmission lines $\mathcal{L}, F \in \mathbb{R}^{N_{l} \times 1}$.

\section{INTRODUCTION}

Nowadays, wind energy is considered as one of the fastest growing renewable energy resources in the world. According to the report of European Wind Energy Association (EWEA), the wind energy should meet $15.7 \%$ of electricity consumption by 2020 and $28.5 \%$ by 2030 [1].

The modern Day Ahead (DA) and Real Time (RT) power market operation with wind power integration is introduced in [2], [3]. In the DA scheduling, the unit commitment problem is assumed to be resolved. In the RT market, short-term wind power production forecasts are submitted to the system operator. The Economic Dispatch (ED) is then executed in order to schedule the dispatchable conventional generators. The interval between two EDs is normally $5 \mathrm{~min}$, according to [3]. During this interval, the wind power fluctuations which are not taken care of by the dispatch signals are handled by the ancillary service-Automatic Generation Control (AGC).

With high wind power penetration level, more operating reserves are required and the ancillary service cost increases. In addition, due to the ramp rate limitation of conventional 
generators, fast wind power fluctuations can hardly be compensated within a short period.

Due to the capabilities of flexible charging-discharging and fast response, Energy Storage System (ESS), especially Battery Energy Storage System (BESS), can participate in AGC to mitigate the fluctuation. Thus, the installation of a minimum of ESS at network buses where power injection has a significant impact on the network is highly desired, which is related to the optimal siting and sizing problems.

For the studies on optimal siting problem, many references suggested the on-site installation of ESS units within Wind Farms (WFs). To be specific, ESS can be either placed at Point of Common Coupling (PCC) or equipped with Wind Turbine Generators (WTGs). It has been shown that the potential siting of ESS can also be at other buses in the power system for the extra benefits, such as deferring or avoiding capacity and transmission upgrade, reduced transmission and distribution losses and more robust system stability [4]-[6].

For the studies on optimal sizing problem, many methodologies have been proposed [7]-[9]. The sizing problem can be modeled as an optimization problem. The related factors were quantified and taken into the cost function, including wind power forecasting accuracy, application purpose, control strategy and economical aspect. However, these studies mainly forcused on the ESS sizing for daily wind power dispatch and the network constraints were not explicitly considered.

In this paper, algorithms for optimal siting ans sizing of ESS are proposed for mitigation of the wind power fluctuation during the interval between EDs. Operations are incorporated via optimal charge-discharge scheduling of the storage units, while respecting the physical network constraints. With various wind generation portfolios, the statistics on the storage activities can be built up, which is used to decide optimal siting and sizing of ESS.

\section{Methodology for optimal Siting AND SiZing}

The flow chart of the proposed optimal siting and sizing algorithms is shown in Fig. 1. The algorithms are interdependent and implemented sequentially.

As the input of the algorithms, long-term Wind Power Time Series (WPTS) can be either the historical wind data records or synthetic data generated by parameterized time series model. For the latter case, various techniques have been developed, such as Markov Chain and Auto Regressive Moving Average (ARMA) models [10]. Since multiple wind farms are integrated, the spatial dependence among different wind sits should also be taken into consideration. In [11], a Copula-ARMA model for wind generation is proposed. In this model, the spatial dependence is modeled by Copula method while the temporal dependence is modeled by ARMA.

\section{A. Optimal siting algorithm}

The optimal siting algorithm is firstly implemented. The derived WPTS is divided into $N$ cycles according to the ED frequency. In this way, various portfolios of wind fluctuations and penetration levels are included. In this study, the length of

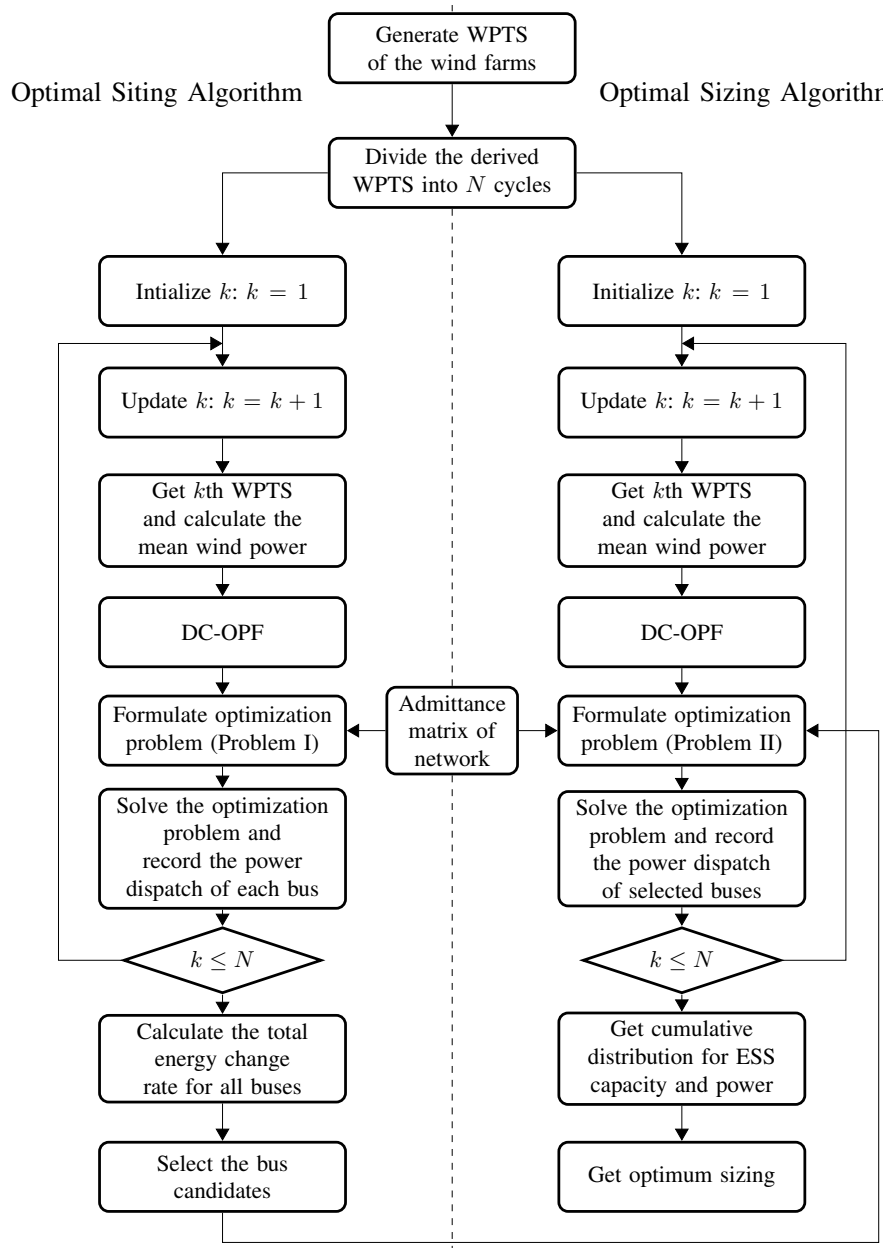

Fig. 1. Flow chart of optimal siting and sizing algorithms

a single cycle is 5 minutes which corresponds to the chargingdischarging cycle of the ESS as well as the interval between two EDs.

For each cycle, the mean wind power forecast is assumed to be perfect. The dispatch of conventional generators can be decided by solving an ED problem. The classical ED problem doesn't consider the network constraints. Instead, the Network-Constrained ED is applied in this study, which could be considered as a special case of the OPF problem. In order to reduce the computation complexity, DC-OPF is used. From the system operator point of view, the participation of the ESS in AGC service can reduce the service cost and improve the regulation capability for the fast variation.

In this algorithm, all the buses are assumed with unlimited amounts of power and energy of ESS units. The chargedischarge control of ESS units can be decided by solving an optimal problem, labeled as Problem I. Accordingly, an absolute ESS power at each bus can be calculated, which is used to evaluate the activity of power exchange for this cycle.

After $N$ cycles, the statistics on the ESS activities at all buses can be summarized. These buses can be ranked according to the power exchanges. A bus with larger power 
exchange indicates that the grid can benefit more if this bus is equipped with ESS. The number of the selected buses for ESS installaion can be decided by the system operator.

\section{B. Optimal sizing algorithm}

Based on the selected buses obtained from the optimal siting algorithm, the control of ESS units for charging and discharging can be decided by solving an optimal problem (labeled as Problem II). After $N$ cycles, Empirical Cumulative Distribution Functions (ECDFs) for power and capacity can be built up, respectively. With a given probability value, which is assumed to fulfill the requirements for sufficient time periods ( $95 \%$ in this study), the optimal sizing can be determined based on the ECDFs.

\section{CYCLIC OPTIMIZATION PROBLEM}

In this section, the cyclic optimization problems for both optimal siting (Problem I) and optimal sizing (Problem II) are formulated.

\section{A. System description}

Consider a power network with $N_{b}$ buses, $N_{l}$ lines, $N_{g}$ generators and $N_{s}$ ESS units. According to the different generator types, $\mathcal{G}$ are classified as follows,

- Conventional generator buses $\mathcal{G}_{g}$ whose generators are dispatchable and committed for the AGC service.

- Renewable energy generator buses $\mathcal{G}_{r}$ whose generators are considered non-dispatchable to make full use of renewable energy.

To be noticed, the set of ESS buses $\mathcal{S}$ is dependent on the optimal algorithms. For the optimal siting algorithm, the ESS units are assumed to be installed at all buses, i.e. $\mathcal{S}=\mathcal{N}$. For the optimal sizing algorithm, the ESS units are installed at selected buses, i.e. $\mathcal{S} \subset \mathcal{N}$.

\section{B. Formulation of Problem I}

1) Cost function: Three terms are considered in the cost function for the optimal siting problem. Suppose the optimization cycle has $M$ steps, the cost function is expressed by,

$$
\min _{p_{i}^{s}, i \in \mathcal{S}} \sum_{k=1}^{M}(\underbrace{\alpha_{1} \sum_{i \in \mathcal{S}} \mid P_{i}^{s}(k)}_{\text {Term } 1} \mid+\underbrace{\alpha_{2} P_{\text {loss }}}_{\text {Term } 2}+\underbrace{\alpha_{3 i} \Delta P_{i}^{g}(k)}_{\text {Term } 3}) .
$$

\section{Term 1: Minimization of the ESS cost}

Frequent charging and discharging can significantly shorten the life of ESS. In this paper, the usage of the ESS is quantified by the absolute summary of power inflow and outflow during the optimization cycle. Here, $\alpha_{1}$ in (1) denotes the weighting cost of total exchanged ESS power.

Term 2: Minimization of transmission losses

The transmission losses of the whole network in DC load flow $P_{\text {loss }}$ can be calculated approximately by,

$$
P_{\text {loss }}=\left(B^{-1} P\right)^{\prime} G\left(B^{-1} P\right) .
$$

where $G \in \mathcal{R}^{N_{b} \times N_{b}}$ with matrix element $G_{i i}=\sum_{j, j \neq i} g_{i j}$, $G_{i j}=G_{j i}=-g_{i j}$. Here, $\alpha_{2}$ in (1) denotes the weighting cost of transmission losses.

Term 3: Minimization of the additional cost by adjusting the conventional generator

In the AGC market, committed generators are capable of providing ancillary services, such as AGC dispatched by the system operator. The dispatched power from these generators lead to an additional cost. With the assumption that the dependence is proportional, the cost can be calculated according to $\alpha_{3 i} \Delta P_{i}^{g}(k)$, where $\alpha_{3 i}$ denotes the weighting cost of the $i$ th generator.

2) Constraints: The constraints related to dispatchable generators, ESS and transmission capacity are listed as follows.

Constraints of dispatchable generators

The deviation of the total wind power production at time $k$ from the predicted mean value of wind power during the cycle is denoted by $\Delta P^{r}(k)$, which can be obtained by,

$$
\Delta P^{r}(k)=\sum_{i \in \mathcal{G}_{r}} P_{i}^{r}(k)-\frac{1}{M} \sum_{k=1}^{M} P_{i}^{r}(k) .
$$

In order to keep the balance between power production and consumption, $\Delta P^{r}(k)$ should be compensated by AGC through the regulation of the ESS units and committed dispatchable generators. The dispatch algorithm between these generators are assumed to be proportional to their power ratings. Let $d_{i}^{g}\left(i \in \mathcal{G}_{g}\right)$ represent the dispatch factor, $P_{i}^{g}(k)$ can be derived,

$$
P_{i}^{g}(k)=P_{i}^{g}(k-1)-d_{i}^{g}\left(\Delta P^{r}(k)+\sum_{i \in \mathcal{S}} P_{i}^{s}(k)\right), \forall i \in \mathcal{G}_{g} .
$$

Accordingly, the power constraint is expressed by,

$$
\underline{P_{i}^{g}} \leq P_{i}^{g}(k) \leq \overline{P_{i}^{g}}, \forall i \in \mathcal{G}_{g},
$$

where $P_{i}^{g} \cdot \overline{P_{i}^{g}}$ denote the lower and upper limitation of $P_{i}^{g}(k)$, respectively.

The power ramp rate constraint is expressed by,

$$
\underline{\Delta P_{i}^{g}} \leq \Delta P_{i}^{g}(k) \leq \overline{\Delta P_{i}^{g}}, \forall i \in \mathcal{G}_{g}
$$

where $\Delta P_{i}^{g}(k)=P_{i}^{g}(k)-P_{i}^{g}(k-1), \Delta P_{i}^{g}, \overline{\Delta P_{i}^{g}}$ denote the lower and upper limitation of $\Delta P_{i}^{g}(k)$, respectively.

Constraints of ESS

In Problem I, ESS units are assumed to be installed at all the buses with unlimited amounts of power and energy. The dispatch period of the ESS corresponds to the chargedischarging cycle of ESS, i.e.,

$$
\sum_{k=1}^{M} P_{i}^{s}(k)=0, \forall i \in \mathcal{S}
$$

Constraints of transmission capacity

The line flows $F$ for all the lines $\mathcal{L}$ are calculated by the following steps.

Firstly, the active power injections $P_{i}(k)$ for all the buses $\mathcal{N}$ are calculated according to the different bus types. 
For the dispatchable generator buses $\mathcal{G}_{g}$,

$$
P_{i}(k)=P_{i}^{g}+P_{i}^{s}-P_{i}^{l}, i \in \mathcal{G}_{g} .
$$

For the renewable energy buses $\mathcal{G}_{r}$,

$$
P_{i}(k)=P_{i}^{r}+P_{i}^{s}-P_{i}^{l}, i \in \mathcal{G}_{r} .
$$

For other buses (load with ESS),

$$
P_{i}(k)=P_{i}^{s}-P_{i}^{l}, i \in \mathcal{L} .
$$

Secondly, DC power flow equations can be expressed in the following matrix form,

$$
F=(b A) B^{-1} P,
$$

where $b \in \mathbb{R}^{N_{l} \times N_{l}}$ is the matrix whose diagonal elements $b_{k k}$ equal to the susceptance of the $k$ th line and the non-diagonal elements are zero. $A \in \mathbb{R}^{N_{l} \times N_{b}}$ is the bus-line incidence matrix, whose element $a_{i j}=1$, if the line exists from Bus $i$ to Bus $j$. For the starting and ending buses, the elements are 1 and -1 , respectively, otherwise $a_{i j}=0$.

Based on (8)-(11), the power flow constraint can be expressed in the matrix form,

$$
\underline{F} \leq(b A) B^{-1} P \leq \bar{F},
$$

where $\underline{F} \in \mathbb{R}^{N_{l} \times 1}$ and $\bar{F} \in \mathbb{R}^{N_{l} \times 1}$ are the vector of upper and lower limits of line flows for all the lines $\mathcal{L}$, respectively.

\section{Formulation of Problem II}

1) Cost function: The cost function of Problem II is identical with that of Problem I.

2) Constraints: The constraints of dispatchable generators, transmission capacity of Problem II are same as these of Problem I. For the ESS constraints, the ESS units are installed at selected buses in Problem II. Therefore, the power and power ramp rate of ESS units at selected buses are limited. Besides, the total power and energy capacity are limited due to the practical constraints. In the following, the additional constraints are listed.

The power and ramp rate constraints are

$$
\begin{array}{r}
\frac{P_{i}^{s} \leq P_{i}^{s}(k) \leq \overline{P_{i}^{s}}, \forall i \in \mathcal{S},}{\Delta P_{i}^{s} \leq \Delta P_{i}^{s}(k) \leq \overline{\Delta P_{i}^{s}}, \forall i \in \mathcal{S} .}
\end{array}
$$

where $P_{i}^{s}, \overline{P_{i}^{s}}$ denote the lower and upper limitation of $P_{i}^{s}(k)$, respectively; $\Delta P_{i}^{s}, \overline{\Delta P_{i}^{s}}$ denote the lower and upper limitation of $\Delta P_{i}^{s}(k)$, respectively.

The total energy capacity constraint is

$$
\sum_{i \in \mathcal{S}}\left|\sum_{k=1}^{M} P_{i}^{s}(k) \Delta t\right| \leq E, \forall i \in \mathcal{S},
$$

where $\Delta t$ indicates the interval between two steps, $E$ is the total energy capacity limit.

\section{Simulation}

In this paper, the IEEE 14 bus system was used as the test system to demonstrate the developed algorithms. The algorithms were implemented in YALMIP [12] with the solver SeDuMi [13]. As illustrated in Fig. 2, two wind farms are included in the system, located at Bus 6 and Bus 8 and termed as WF 1 and WF 2, respectively.

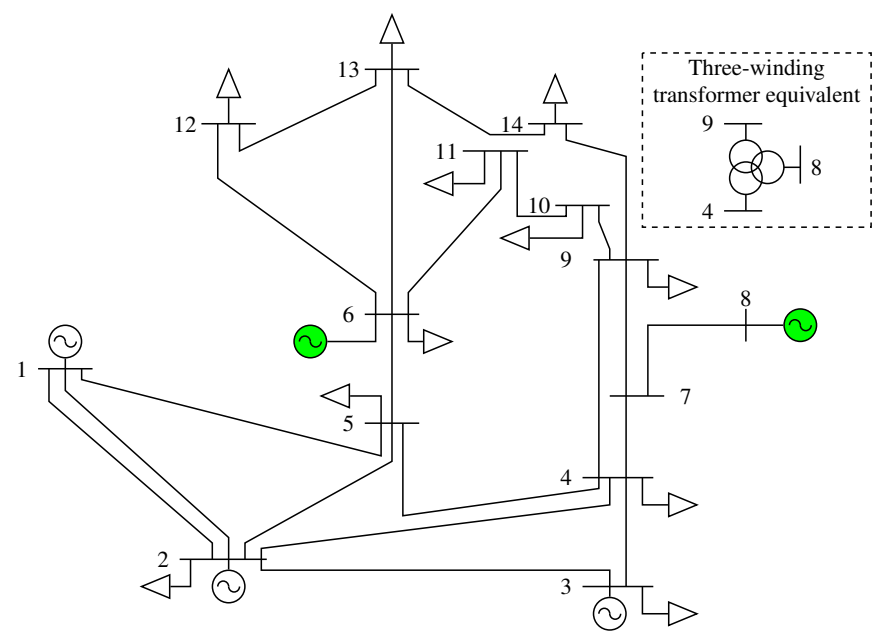

Fig. 2. Single line diagram of IEEE 14 buses system

\section{A. Optimal Siting}

The optimal siting of ESS is dependent on many factors, including the wind power fluctuation, transmission capacity and etc. By taking these factors into account for various realizations of wind power generation, the power exchanges for all the buses can be derived, as illustrated in Fig. 3. Their ranking can be considered as the criterion for the bus selection.

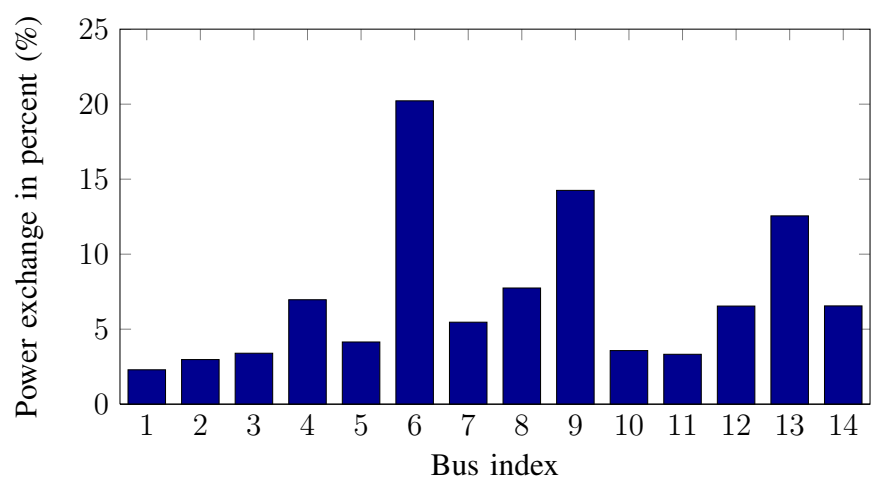

Fig. 3. Power exchange in percent

In order to investigate the influence of transmission capacity on the ESS siting, the transmission capacity between Bus 13 and Bus 14 has a tighter limitation (6.5 MW). Obviously, Buses 6, 9 and 13 have the highest power exchanges and they are hereby selected for ESS installation. For Bus 6 and 9, it can be understood that if most wind power fluctuations are compensated on site, the transmission losses will be reduced. 
For Bus 13, it can be understood that in order to avoid overloading of the line with limited transmission capacity, the ESS unit is preferred to be installed at the terminal bus of the line.

\section{B. Optimal Sizing}

For the power system with multiple wind farm integration, the correlation between wind farms has a significant impact on the ESS sizing. In order to investigate to which extent the correlation of WF 1 and WF 2 affects the power and capacity ratings, two case scenarios are defined in Table I. Pearson's $r$ is used to represent the linear correlation between WF 1 and WF 2. Smaller value indicates the more obvious smoothing effect of multiple wind farms. In other words, wind power fluctuation is reduced from the system point of view.

TABLE I

CASE SCENARIO DEFINITION

\begin{tabular}{c|c}
\hline Scenario & Pearson's $r$ \\
\hline \hline Scenario 1 & 0.22 \\
\hline Scenario 2 & 1.00 \\
\hline
\end{tabular}

For the power rating, $F_{\text {pow }}$ is defined as the Empirical CDF (ECDF) of power and $F_{\text {pow }}^{-1}$ is the inverse of $F_{\text {pow }} \cdot F_{\text {pow }}^{-1}(0.95)$ represents the guaranteed power rating requirement for $95 \%$ of the time. Considering the technical limitation, the upper limits of all ESS units are set as $\overline{P_{i}^{s}}=3 \mathrm{MW}$ in this study. The power ratings of all the selected buses (Buses 6, 9, 13) for both scenarios are almost the upper limits: $3 \mathrm{MW}$.

For the energy capacity rating, $F_{\text {cap }}$ is defined as the ECDF of capacity and $F_{\text {cap }}^{-1}$ is the inverse of $F_{\text {cap }} . F_{\text {cap }}$ of all the selected buses (Buses 6, 9, 13) is illustrated in Fig. 4 and $F_{\text {cap }}^{-1}(0.95)$ are listed in Table II. It can be observed that for the selected buses, larger energy capacities are required to compensate the larger power fluctuations in Scenario 2, compared with these in Scenario 1. The total required energy capacity in Scenario 2 is $1.64 \mathrm{MWh}$ which is $18.0 \%$ larger than that of Scenario $1(1.39 \mathrm{MWh})$. Thus, the correlation between wind farms has a significant impact on the ESS sizing.

TABLE II

ENERGY COMPARISON

\begin{tabular}{l|l|l}
\hline Bus index & $\begin{array}{l}F_{\text {cap }}^{-1}(0.95) \\
\text { (Scenario 1) }\end{array}$ & $\begin{array}{l}F_{\text {cap }}^{-1}(0.95) \\
\text { Scenario 2) }\end{array}$ \\
\hline \hline Bus 6 & $0.52 \mathrm{MWh}$ & $0.61 \mathrm{MWh}$ \\
\hline Bus 9 & $0.54 \mathrm{MWh}$ & $0.59 \mathrm{MWh}$ \\
\hline Bus 13 & $0.33 \mathrm{MWh}$ & $0.44 \mathrm{MWh}$ \\
\hline \hline Summary & $1.39 \mathrm{MWh}$ & $1.64 \mathrm{MWh}$ \\
\hline
\end{tabular}

\section{CONCLUSION}

In this paper, the algorithms for optimal siting and sizing of ESS in the grid with high wind power penetration are presented. For optimal siting algorithm, all the buses are assumed to have ESS installation with unlimited amounts of (a)

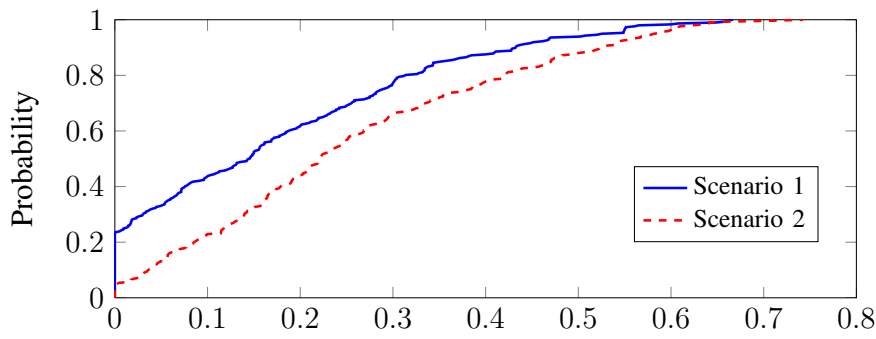

(b)

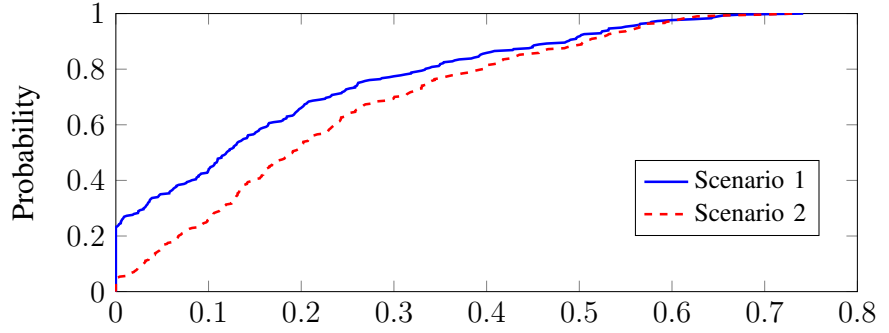

(c)

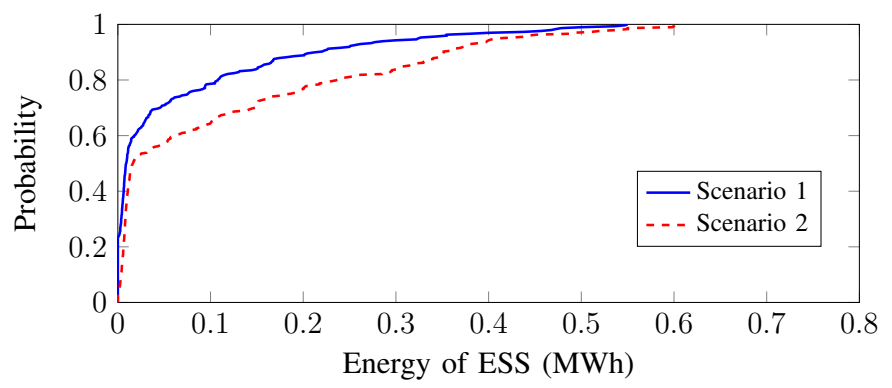

Fig. 4. $F_{\text {cap }}$ at selected buses, (a) Bus 6, (b) Bus 9, (c) Bus 13 .

power and energy. The absolute power exchange of ESS at each bus is used to evaluate its activity. The activity ranking of all the buses can be used as the criterion for the optimal siting. It can be concluded that the placements of ESS include not only the wind farm sites, but also other buses, such as terminal bus of critical transmission line. For optimal sizing algorithm, the additional power and energy constraints are incorporated in the optimization problem. By solving a large number of cyclic optimization problems, the ECDFs of power and energy capacity of ESS for different realizations of wind power fluctuations are obtained. Accordingly, the optimal power and energy rating can be determined by setting the probability level (95\% in this study). It shows that the correlation between wind farms has a significant impact on the power and energy rating.

\section{ACKNOWLEDGMENT}

The authors gratefully acknowledge the financial support of Sino-Danish Center for Education and Research to the $\mathrm{Ph} . \mathrm{D}$ project "Coordinated Control of Wind Power Plants and Energy Storage Systems".

\section{REFERENCES}

[1] J. Moccia, A. Arapogianni, et al., "Pure power. wind energy targets for 2020 and 2030," EWEA, 2011. 
[2] Q. Li, S. Choi, Y. Yuan, and D. Yao, "On the determination of battery energy storage capacity and short-term power dispatch of a wind farm," IEEE Trans. Sustain. Energy, vol. 2, no. 2, pp. 148-158, 2011.

[3] A. Botterud, J. Wang, C. Monteiro, and V. Miranda, "Wind power forecasting and electricity market operations," Proceedings of USAEE, vol. 3, p. 3846, 2009.

[4] S. Tewari and N. Mohan, "Value of NAS Energy Storage Toward Integrating Wind: Results From the Wind to Battery Project," IEEE Trans. on Power Syst., vol. 28, no. 1, pp. 532-541, Feb. 2012.

[5] M. Ghofrani, A. Arabali, M. Etezadi-Amoli, and M. Fadali, "A Framework for Optimal Placement of Energy Storage Units Within a Power System With High Wind Penetration," IEEE Trans. Sustain. Energy, vol. 4, no. 2, pp. 434-442, 2012.

[6] S. Bose, D. F. Gayme, U. Topcu, and K. M. Chandy, "Optimal placement of energy storage in the grid," in Decision and Control (CDC), 2012 IEEE 51st Annual Conference on. IEEE, 2012, pp. 5605-5612.

[7] J. P. Barton and D. G. Infield, "A probabilistic method for calculating the usefulness of a store with finite energy capacity for smoothing electricity generation from wind and solar power," Journal of Power Sources, vol. 162, no. 2, pp. 943-948, Nov. 2006.

[8] H. Bludszuweit, "Statistical analysis of wind power forecast error," IEEE Trans. Power Syst., vol. 23, no. 3, pp. 983-991, 2008.

[9] H. Bludszuweit and J. A. Domínguez-navarro, "A probabilistic method for energy storage sizing based on wind power forecast uncertainty," IEEE Trans. Power Syst., vol. 26, no. 3, pp. 1651-1658, 2011.

[10] P. Chen, T. Pedersen, B. Bak-Jensen, and Z. Chen, "Arima-based time series model of stochastic wind power generation," IEEE Trans. Power Syst., vol. 25, no. 2, pp. 667-676, 2010.

[11] Y. Li, K. Xie, and B. Hu, "Copula-arma model for multivariate wind speed and its applications in reliability assessment of generating systems," Journal of Electrical Engineering \& Technology, vol. 8, no. 3, pp. 421-427, 2013.

[12] J. Lofberg, "Yalmip: A toolbox for modeling and optimization in matlab," 2004 IEEE International Symposium on Computer Aided Control Systems Design, IEEE, 2004, pp. 284-289.

[13] J. F. Sturm, "Implementation of interior point methods for mixed semidefinite and second order cone optimization problems," Optimization Methods and Software, vol. 17, no. 6, pp. 1105-1154, 2002. 